

Appareil

12 | 2013

Walter Benjamin. Politiques de l'image

\title{
L'innervation gestuelle-et-politique de la perception et du cinéma
}

\section{Philippe Roy}

\section{(2) OpenEdition}

1 Journals

Édition électronique

URL : https://journals.openedition.org/appareil/1939

DOI : 10.4000/appareil.1939

ISSN : 2101-0714

Éditeur

MSH Paris Nord

Référence électronique

Philippe Roy, "L'innervation gestuelle-et-politique de la perception et du cinéma », Appareil [En ligne], 12 | 2013, mis en ligne le 13 décembre 2013, consulté le 21 septembre 2021. URL : http:// journals.openedition.org/appareil/1939; DOI : https://doi.org/10.4000/appareil.1939

Ce document a été généré automatiquement le 21 septembre 2021.



Appareil est mis à disposition selon les termes de la Licence Creative Commons Attribution - Pas d'Utilisation Commerciale - Pas de Modification 4.0 International. 


\title{
L'innervation gestuelle-et-politique de la perception et du cinéma
}

\author{
Philippe Roy
}

\section{L'innervation gestuelle de la perception}

\subsection{Puissance des gestes}

1 En parlant d'innervation gestuelle de la perception, je voudrais dire pour commencer que je n'entends pas simplement le fait que la perception est subordonnée à l'action. En disant par exemple que je perçois ce stylo parce que je veux écrire. Action qui elle-même répondrait à un besoin, donc à la vie. C'est même contre ce rapport simpliste que j'irai. Cette introduction de la vie dans l'élément de la perception a été considérée un temps comme un progrès, s'opposant à une perception trop désincarnée, liée soit à des données sensibles atomiques répondant à des principes d'association propres à l'entendement (les empiristes), soit à une idée unifiante, elle-même métonymie du sujet pensant, comme le montre Descartes dans son analyse du morceau de cire (position intellectualiste).

2 Ce qu'il y a de commun à l'empirisme et à l'intellectualisme est qu'ils supposent une coordination de l'unité d'un sujet pensant et d'unités sensibles (les données sensibles) de la chose perçue. Et au fond, c'est l'intellectualisme qui est la vérité des deux, l'unité des données sensibles n'étant que la projection de l'unité du sujet pensant (c'est lui qui " atomise »). La question se posant cependant de savoir si la conscience de ces unités ne viendrait pas de la réflexion de la conscience sur le perçu déjà constitué, et en ce sens les unités ne seraient pas constituantes de la perception car elles seraient des unités propres à la réflexion et non à la perception.

3 Autre problème, lié au précédent: il n'est pas pris en compte comment le sujet se rapporte au perçu. Pour éclairer ce rapport, Husserl rectifiera les choses en montrant l'existence d'actes intentionnels, mais sans remettre en cause l'existence de ces unités auxquelles ces actes se rapportent, unités des données sensibles de la hylè (les vécus) 
esquissant l'unité de la chose visée par l'acte intentionnel, chose toujours à l'horizon. Husserl conserve les prérequis de la conscience réflexive.

Or, si unités il y a dans la perception, n'est-ce pas parce qu'elles sont les arêtes qui composent le monde d'un être vivant, pour qui elles ont une signification. Comme l'a montré l'éthologue Uexküll, cette odeur de mammifère est perçue par la tique car elle est le signal qui va lui faire déclencher une action, qui est celle de se laisser tomber sur l'animal pour ensuite se lancer dans l'exploration tactile, pour au bout du compte pomper le sang et nourrir sa progéniture. Cependant, comment expliquer cet enchaînement d'une perception et d'une action, puis d'une autre perception et d'une autre action, etc. ? Remarquons que cette distinction entre perception et action est déjà abstraite car une action n'est pas sans être elle-même perçue, il y a une perception de l'action et des perceptions propres à l'action, ce que Berthoz range sous le nom de «sens du mouvement »: la proprioception (sens de la position et de la vitesse, du toucher, de l'effort et de tout ce qui renvoie au système vestibulaire de l'équilibre, de la rotation, de l'accélération).

5 Alors on dira que c'est le besoin, ici celui de se reproduire, qui conditionne cet enchaînement. Le sang du mammifère est l'objet qui vient répondre au besoin de se reproduire de la tique. Comme si l'enchaînement n'était qu'accessoire. Or c'est ceci qui, je crois, pose problème, car l'objet est inséparable de cet enchainement, la tique ne veut le sang que parce qu'elle possède cet enchaînement, l'objet s'est constitué originellement avec lui. Cet enchaînement qui mêle perceptions et actions, je l'appelle " un acte » et un acte est un geste, même si un geste ne se réduit pas à être un acte, j'y reviendrai. La tique effectue un geste qui com-prend le sang en fin de geste. Le geste s'effectue entre la tique et le sang, le geste est ici relationnel.

6 On le comprendra mieux si est rectifiée une formulation imparfaite : à vrai dire la tique n'effectue pas un geste, la tique n'est pas autre chose que ce geste, il ne faut pas distinguer un sujet et un acte, comme Nietzsche l'a montré dans Généalogie de la morale ${ }^{1}$, le prédateur n'est pas séparable de son geste qui com-prend sa proie. On ne peut détacher, sinon verbalement, le sujet, le geste et l'objet. Tout est affaire de puissances d'agir, de ce que l'on peut, et la tique peut ce geste qui la rapporte au sang. La tique est constituée par ses gestes et non constitutives d'eux, ce pourquoi l'individu s'efface derrière ses gestes et que ceux-ci se poursuivront avec sa progéniture. Les actes, qui sont toujours ceux d'un ici-maintenant, supposent une part virtuelle, qui est, ce qui du geste, conditionne sa répétition : la part virtuelle du geste. De l'acte au geste virtuel, il y a une boucle, un circuit qui relie chaque tique effective au geste-événement virtuel qu'a été le premier enchaînement jusqu'au sang et qui est maintenant celui de l'espèce.

7 Le besoin du sang est l'affect qui correspond, pour la tique, au geste qui veut s'effectuer entre elle et le sang. Ce geste qui, en étant effectué, augmentera sa puissance. On retrouve donc ici des accents nietzschéens et spinozistes. Attardons-nous alors sur la conception gestuelle de l'individualité de Spinoza. Il définit ainsi ce qu'il appelle «Individu » dans le livre 2 de l'Éthique:

Quand certains corps de même grandeur ou de grandeur différente sont contraints par les autres corps à rester appliqués les uns contre les autres ou, s'ils se meuvent à la même vitesse ou à une vitesse différente, sont contraints à se communiquer leur mouvement les uns aux autres selon un certain rapport, nous disons que ces corps sont unis entre eux et que tous composent ensemble un seul corps, c'est-àdire un Individu qui se distingue des autres par cette union des corps ${ }^{2}$. 
Cette définition peut paraître au départ effroyablement abstraite. Essayons de l'illustrer dans le cas d'un individu humain. Ma main qui bouge contraint mes doigts à bouger avec une même vitesse par rapport au reste de mon corps et elle communique son mouvement de rotation à mon avant-bras et est contrainte par le bras à ne pas faire une rotation complète. On voit qu'en essayant d'illustrer cette définition, c'est une certaine aptitude gestuelle qui se présente. C'est un geste visible. Mais l'individualité inclut aussi tous les gestes invisibles associés aux rapports des parties invisibles de mon corps, même les cellules ont leurs gestes... Or ces gestes, en tant qu'ils appartiennent à une individualité, doivent pouvoir permettre leur répétition, il faut une réversibilité des gestes, si je peux tourner ma main dans un sens, je dois pouvoir la tourner dans le sens opposé, pour pouvoir permettre la répétition du premier geste. Bien plus, chaque geste doit permettre la répétition de tous les autres gestes de cette même individualité, se gratter la tête est un geste qui n'a pas d'incidence sur la répétition des autres (il est mien), par contre sauter d'une falaise risque de m'en faire perdre plus d'un... Il n'est pas mien (du moins tant que je n'en suis pas capable sans en éliminer d'autres). Se nourrir est un geste pour la répétition des autres gestes, pour que notre puissance d'agir se déploie. Ce pourquoi la position vitaliste est extrêmement réductrice. On ne vit pas que pour ses besoins mais pour effectuer des gestes. Observons un animal: combien de gestes faudrait-il lui retirer si on ne conservait que ceux qu'il effectue pour sa conservation? Caractériser un individu par des gestes ne veut pas dire non plus qu'il n'y a pas de gestes communs à deux ou plusieurs individus. Même s'il est difficile d'entendre le terme de geste appliqué au sang (car il est invisible), il n'empêche que le sang est composé de corps qui sont dans un certain rapport de mouvements et de repos qui forment aussi un geste invisible possible de la tique. Assimiler le sang, c'est effectuer ce geste du sang qui fait partie des siens. Sous la perception du sang par la tique, il y a donc un geste, celui-ci commande sa perception, il l'innerve.

9 Devenir actif, c'est développer ses aptitudes ou même des aptitudes plus qu'individuelles quand elles se composent passagèrement ou plus longuement avec un autre individu. Comme lorsque l'on fait usage d'un objet technique qui nous donne momentanément une aptitude supplémentaire, qui ne nous est donc pas attribuable en propre. Nous et l'objet technique formons un individu plus grand (par exemple quand nous faisons corps avec notre voiture). Cette composition peut aussi se faire entre gestes différents. Quand je bricole avec quelqu'un, nos gestes doivent concorder. Un affect en découle: quand cela concorde, j'augmente ma puissance (joie), sinon je la diminue (tristesse). Et justement, dans le cas où il y a diminution de puissance, je me retrouve sans geste, confronté à un enchaînement désordonné de perceptions et d'actions, une sensori-motricité qui cherche son geste. En terme spinoziste, je ne suis plus cause adéquate de ce que je fais, je ne suis plus ce geste. Perception et action ne sont plus innervées par un geste. Et on comprend aussi que plus je possèderai de gestes, plus je serai à même de réagir à des situations subies, sans me laisser entraîner dans une sensori-motricité désordonnée. Plus un individu est actif, moins il subit ${ }^{3}$. L'étymologie du terme de geste vient de gero qui veut dire " porter » car nous avons à être des gestes, à en être les porteurs. Sinon nous ne sommes que des causes partielles de ce qui nous arrive.

10 Ce pourquoi Yves Citton dans Renverser l'insoutenable peut écrire: "Qu'un camion me percute et me projette du haut d'un pont, qu'un ami soulève ma jambe paralysée pour la placer sur un coussin, on ne me créditera pas d'un "geste", puisque ces mouvements 
(de mon corps) ne seront pas déclenchés par une circulation interne à mon corps ${ }^{4} »$. Je ne suis pas cause adéquate de ce qui m'arrive. Si bien que l'on peut dire que la perception de quelque chose avec laquelle nous n'allons pas pouvoir composer gestuellement doit induire un affect de tristesse, par exemple de " peur » (comme celle liée à ce camion qui me fonce dessus), et à l'inverse, la perception associée à une joie est le gage d'une composition gestuelle. Si bien que l'affect même montre que la perception est d'emblée gestualisée, dans la ligne de mire d'un geste. L'affect manifeste ici des rapports gestuels et donc, au fond, des rapports de puissances individuelles.

\subsection{L'innervation gestuelle au regard de la neurophysiologie}

11 Les recherches actuelles de neurophysiologie confirment cette innervation gestuelle de la perception. J'en donne un aperçu. Alain Berthoz et Jean-Luc Petit dans Phénoménologie et physiologie de l'action évoquent le geste de monter les escaliers qui, dès la perception des escaliers, implique une activité des zones motrices du cerveau en même temps que les zones sensorielles :

La perception est structurée par l'interaction de l'agent avec le monde [...] l'évaluation de la hauteur d'une marche d'escalier par le cerveau de celui qui veut le gravir n'est pas faite en en mesurant la hauteur en centimètres [...] L'évaluation en question est plutôt celle du rapport entre la hauteur perçue de la marche et la hauteur perçue du pas du sujet ${ }^{5}$.

C'est notre geste de monter les escaliers qui innerve notre perception des escaliers. Pensons aussi à la théorie des affordances de Gibson (Approche écologique de la perception visuelle), "celui-ci avait noté que, des objets visuels environnants, nous percevons surtout les ressources pratiques qu'ils comportent pour l'action ${ }^{6}$ ». La perception d'une cuillère enveloppe le geste de touiller.

Il y a une simulation gestuelle impliquée par la perception. En quoi on voit bien que le geste ne se réduit pas à l'acte, c'est-à-dire au geste effectué. Des neurones (dit canoniques) s'activent à la fois lors de l'exécution d'un geste et lors de la perception d'un objet saisissable par ce geste ${ }^{7}$. Gérard Olivier, dans son livre La Cognition gestuelle, peut donc écrire «la perception visuelle d'un objet saisissable s'accompagne de la simulation inconsciente de l'atteinte et de la saisie manuelle de cet objet ». On retrouve bien ici ce que je disais plus haut avec la tique, l'objet est inséparable de son geste. Dès le début de l'enchaînement, le geste invisible du sang innerve la perception de la sueur du mammifère, cette innervation s'accompagnant aussi du geste qui permet d'atteindre le sang, c'est-à-dire le geste de l'enchaînement.

C'est cette simulation inconsciente qui ne pouvait être qu'occultée par les approches de la perception telle que l'empirisme, l'intellectualisme dont j'ai parlé plus haut, centrées, elles, sur la conscience. Or, même dans ces approches qui pointent des données sensibles, des choses visées, il y a un geste impliqué qui est celui de la désignation, le geste de désigner, de montrer. Ce geste est en effet impliqué par les perceptions soi-disant pures, il ne se réduit pas à l'acte de montrer du doigt, il est impliqué par toute visée (et donc aussi celle, virtuelle, de l'acte intentionnel husserlien). L'objet désigné est donc inséparable d'un geste, il ne le devance pas. Je désigne cet objet car il est déjà désignable, je le vois comme désignable. La simulation du geste de désigner est impliquée par l'objet perçu désigné, il ne pré-existe pas à ce geste. Il ne faut pas séparer le geste et l'objet, le geste étant bien encore ici entre celui qui l'effectue et l'objet. 
Aussi, toujours dans les recherches de neurophysiologie,

la découverte de neurones plurimodaux (visuels et moteurs; visuels et tactiles...) auxquels il est difficile de n'assigner qu'une seule fonction, soit sensorielle, soit motrice, pourrait être interprétée comme l'indice de l'appartenance de ces neurones à des structures associées ni à un objet ni à un geste particulier, mais à une situation englobant à la fois le geste et l'objet ${ }^{8}$.

16 Il y a bien une innervation gestuelle inconsciente de la perception inséparable de son objet. On pourrait montrer que même la perception de l'espace suppose un geste virtuel (groupe des déplacements de Poincaré), même «l'image visuelle d'un objet est assimilable à l'ébauche de la simulation mentale d'une exploration oculaire de cet objet, et de la même manière, l'image auditive d'un son du langage est assimilable à la simulation mentale de sa prononciation ${ }^{9}$ ». Il faut noter que Benjamin a été très intéressé par les auteurs qui pensent une origine gestuelle du langage, tel Marcel Jousse ou Richard Paget auxquels il consacre plusieurs pages dans son texte «Problèmes de sociologie du langage ». Il écrit :

Ainsi, selon Paget, l'articulation comme geste de l'appareil linguistique se rattache

à l'ensemble de la mimique corporelle. Son élément phonétique est le porteur d'une communication dont le substrat originaire était un geste expressif ${ }^{10}$.

Le geste est donc impliqué aussi par la perception auditive du langage parlé. Le geste est l'inconscient de la perception, notre conscience étant, elle, au service du geste pour en assurer l'effectuation, tournée vers l'objet, signe pour nous de l'objet. Le geste dirige la conscience pour que celle-ci s'applique à l'effectuer et donc en repère les objets d'application. On comprend donc que toute approche de la perception basée sur la conscience est vouée à l'incomplétude. Lorsque je conduis ma voiture, je n'ai pas conscience du geste que j'effectue en faisant corps avec elle, j'ai conscience seulement de ses points d'application, c'est-à-dire par exemple de la route que je dois suivre. La seule conscience de la route évitant d'ailleurs la conscience d'autres actions du geste (je ne me perçois pas en train de conduire) qui pourrait empêcher sa bonne effectuation (on effectue mal un geste si la conscience l'envahit trop). Il n'y a de bon geste que dans un somnambulisme dirigeant la part suffisante de conscience (le signe) pour bien assurer son effectuation. Et c'est parce qu'il y a la part suffisante de conscience que le somnambulisme est assuré. Au point que, comme l'a énoncé Bergson, la conscience s'atténue avec la maîtrise du geste tendant à l'automatisme du somnambule.

\section{Innervations gestuelles du cinéma}

On peut alors se tourner encore, mais plus longuement, vers Benjamin qui, dans "L'œuvre d'art à l'ère de la reproductibilité technique » (première version ${ }^{11}$ ), écrit : "Nous connaissons en gros le geste que nous faisons pour saisir un briquet ou une cuiller, mais nous ignorons à peu près tout du jeu qui se déroule réellement entre la main et le métal, à plus forte raison des changements qu'introduit dans ces gestes la fluctuation de nos diverses humeurs ». Ici, Benjamin distingue bien deux choses : une perception grossière, celle de la conscience pour le geste (dans l'enchaînement perception-action) et celle du geste lui-même, le jeu qui se déroule entre la main et le métal. Or, continue-t-il, c'est dans ce domaine, donc du geste, que pénètre la caméra, « pour la première fois, elle nous ouvre à l'inconscient visuel ». Pour Benjamin, le geste est bien aussi l'inconscient de la perception visuelle (il ne parle cependant pas ici d'innervation, la réservant à l'innervation des nerfs moteurs). Mais pourquoi le cinéma 
aurait cette capacité de pénétrer dans le domaine gestuel ? Parce que les images sont innervées par un certain nombre de gestes cinématographiques: plongées et remontées de la caméra, coupures et isolements, ralentissements et accélérations du mouvement, agrandissements et réductions. Plonger, remonter, couper, isoler, ralentir, accélérer, agrandir, réduire et bien sûr monter (le montage faisant retentir les gestes les uns avec les autres et dans les autres). Ces gestes se donnent avec l'image. Par exemple, «cadrer » est un geste impliqué par l'image cinématographique, ce pourquoi une ombre chinoise n'est pas une image cinématographique puisqu'elle dépend du seul point de vue du spectateur. Le geste est mieux révélé par un film parce que filmer est gestuel. C'est qu'en effet, le cinéma est plus proche de l'enveloppe virtuelle du geste que de son effectuation, en quoi il nous rend attentifs aux gestes.

Il faudrait s'expliquer plus longuement sur cette idée d'enveloppe virtuelle du geste. Je donne seulement un aperçu de cette distinction. Le geste a deux polarités, celle de l'actuel, tournée vers son effectuation, vers son acte et donc les actions-perceptions et les objets, conscience partielle pour le geste. Le deuxième pôle est plutôt du côté de l'unité du geste, ce qui fait de lui un tout, si bien qu'il serait plus du côté de l'exhibition d'ensemble du geste, le geste se montre, il a comme une enveloppe qui vient frapper ceux qui le perçoivent au point que nous mimons ou simulons le geste perçu sans en avoir conscience et sans que cette simulation soit à l'image de la conscience de ce qui est perçu. On a pu détecter l'existence de neurones miroirs s'activant lors de la seule perception visuelle du geste exécuté par un autre, activation identique chez l'agent exécutant le geste. La première polarité va du simulateur au geste effectué (l'acte), la deuxième du geste effectué au simulateur, le geste se virtualise, il vient prendre place en nous sous forme d'un simulateur. Mais ces polarités coexistent, leur distinction vient de deux modalités perceptives. Sous la première modalité, on s'intéresse plutôt à ce que fait le geste (que nous soyons ou pas celui qui l'effectue); sous la deuxième, à sa manière d'être. Je peux saisir le geste de ce footballeur en tant qu'il est celui qui a fait telle action pour tel but (moyen pour une fin) ou alors apprécier ce geste lui-même (moyen sans fin dirait Agamben), et bien mieux encore avec, justement, des caméras disposées sur le bord du terrain.

20 J'ai opté pour le concept de diagramme plutôt que celui de simulateur, car simuler semble laisser croire qu'il y a en nous comme une copie du geste alors que ce sont plutôt des images dynamiques, des intensités et des placements topologiques impliqués par le geste qui composent le diagramme. D'ailleurs, c'est ce que soutiennent aussi les neurophysiologistes, les simulateurs «sont des déroulements potentiels de mouvements cognitifs globaux ${ }^{12}$ ", « des noyaux dynamiques » engageant une activité globale des neurones. Ainsi, dans le cas de la perception de triangles, ce sont des intensités de directions des trois côtés qui composent le noyau dynamique et non des images de ces côtés.

Le noyau dynamique n'a retenu que ce qu'il y a de commun à toutes les explorations passées de triangles : en l'occurrence, un enchaînement de saccades effectuées dans trois directions successives différentes, s'accompagnant d'une faible variation des activations rétiniennes péri-fovéales et tendant à aboutir à un retour au point de départ ${ }^{13}$.

21 On voit ici qu'il est bien question de topologie car le triangle doit se refermer. Et que, de plus, l'ordre chronologique n'est pas primordial, peu importe par quelle direction on commence, ce sont les relations, les rapports différentiels qui importent. Les diagrammes se disent d'un temps non chronologique, du virtuel en tant que passé pur 
qui fait passer les présents, comme Bergson l'a montré. C'est la relativité des mouvements qui compte plus que le renvoi à un repos de référence. Or c'est bien cette relativité que capte l'image cinématographique, impossible de dire si l'image d'un automobiliste dont la caméra est placée dans sa voiture est celle de la caméra en mouvement, via celle de l'automobile, ou si ce sont les images du défilement de la route, via une projection dans un studio...

Un diagramme implique donc des placements et des intensités propres à chaque place et en rapport avec celles des autres places, des intensités liées à des rapports différentiels. Il suppose des places différentes, qui peuvent être celles d'un individu et d'un objet (geste technique) ou de deux ou plusieurs individus (geste technique collectif, geste affectif, geste politique). Chaque geste a son diagramme.

Nous vivons donc inconsciemment une grande part de notre vie en ce niveau diagrammatique, puisque chaque geste effectué suppose un diagramme comme chaque geste seulement perçu ou même pensé. Un diagramme, de par sa réalité intensivevirtuelle, ne peut donc pas se présenter dans le monde sensible massique des perceptions-actions. Le niveau diagrammatique est plutôt celui de la réalité à masse nulle, celui de l'imagination plutôt que de la perception. En effet, imaginer des gestes possibles ou nouveaux a comme condition de possibilité ce niveau diagrammatique. Nous pensons à partir d'un répertoire de diagrammes, de gestes, déposés en nous par l'effectuation de gestes ou l'imagination des diagrammes de gestes, mais aussi par l'exhibition des gestes des autres et la participation à leurs gestes. Nous verrons que participer aux gestes des autres ou avec d'autres est la condition d'une intégration d'une pensée politique sous forme donc de gestes politiques.

Revenons alors au cinéma, celui-ci n'étant fait que d'images-mouvements à masse nulle, comme dans un rêve, il est donc plutôt du côté virtualisant du geste que du côté actualisant. Le cinéma, composé de gestes cinématographiques, ne nous tourne pas du côté de l'acte, de l'effectuation massique. Ce pourquoi il peut jouer de toute la plasticité gestuelle du niveau diagrammatique, et donc explorer gestuellement les gestes sans être emporté par leur application, par l'effectuation. Et c'est selon tel geste cinématographique que chaque geste est exhibé. Le cinéma pénètre donc le réel du niveau diagrammatique, l'inconscient visuel. Ce pourquoi Benjamin peut comparer le caméraman à un chirurgien, leurs tours de main sont de l'ordre du geste ${ }^{14}$. Bien plus, c'est parce que le cinéma se dit de ce niveau diagrammatique imagino-moteur qu'il peut créer des gestes ineffectuables et proposer des déformations, c'est-à-dire des images qui ne sont plus subordonnées à la réalité massique de l'effectuation. Alain Naze est très bien inspiré quand il souligne l'importance du traitement cinématographique de la couleur, qui est libérée de son attachement aux choses massiques, par exemple dans le cinéma d'Eisenstein. Il rappelle que ce dernier, en s'adressant à ses étudiants, leur demandait que le rouge, le noir rompent leur lien initial avec l'objet ${ }^{15}$. Le traitement autonome des couleurs forme un geste au sein de cette matérialité ondulatoire, comme il peut l'être dans la matérialité ondulatoire sonore. Pour conclure sur ce rapport au cinéma, on peut donc dire que le cinéma met en valeur l'innervation gestuelle du visible (mais aussi du sonore). Inversement, puisqu'étant lui-même gestuel, il sera à même par ses gestes (qui sont comme des chocs) d'innerver en retour nos actes et donc nos actions et perceptions. Il nous en incorporera. Citons encore Alain Naze : « Pur mouvement d'images, le film de cinéma ne se laisse pas recueillir comme un bien, et les traces qui sont les siennes sont celles qu'il inscrit en notre corps, par 
innervation ${ }^{16} »$. On comprend donc ici toute la portée politique du cinéma, il révèle des gestes innervant nos perceptions et il innerve aussi nos perceptions. Il peut produire de nouveaux gestes politiques comme en renforcer d'autres par des innervations collectives (qui peuvent autant être celles de la révolution que du totalitarisme). C'est un point sur lequel Yves Citton a mis grandement l'accent dans ses deux livres consacrés aux gestes (Renverser l'insoutenable et Gestes d'humanités ${ }^{17}$ ). Nous sommes placés en immersion gestuelle, d'autant plus avec ces moyens de diffusion que Benjamin repère avec l'avènement de l'ère de la reproductibilité technique, nous sommes à l'âge des ondes.

\section{Innervation gestuelle de la perception en politique}

\subsection{Qu'est-ce qu'un geste politique ?}

Mais qu'est-ce précisément qu'un geste politique ? J'ai beaucoup insisté sur l'aspect relationnel d'un geste, il est entre celui qui l'effectue et l'objet. Il suffit alors de considérer cette fois-ci que le geste est entre deux hommes ou plusieurs et son caractère politique se dévoile. On vient de le voir avec la circulation des gestes, ils ne circulent que parce qu'ils sont entre ceux qui les effectuent et ceux qui les perçoivent. Mais le geste peut être un rapport de force, une action sur une action, ou la conduction d'une autre conduite comme le pense Foucault quand il thématise le pouvoir. Mais entendons bien que le geste n'est pas dans les mains de celui qui exerce l'action, il est entre celui qui l'exerce et celui qui la subit.

Par exemple, le geste de commander suppose autant une certaine conduite du côté de celui qui commande que du côté de celui qui est commandé. Le geste de commander nécessite un certain diagramme de verticalité que doivent activer chacun des acteurs, commandant et commandé. La verticalité suppose le dressement de celui qui ordonne et la position courbée, le regard vers le sol horizontal, chez celui qui reçoit l'ordre. Il y a une perpendicularité virtuelle impliquée par le commandement. Dès qu'il y a commandement, chaque agent prend place en cette perpendicularité, l'agent qui commande est sur la verticale et l'autre sur l'horizontale, mais l'un ne va pas sans l'autre. On ne commande pas à quelqu'un qui se dresse devant vous, et on n'obéit pas à quelqu'un qui vous commande en regardant le sol. J'ajouterai que cette perpendicularité suppose une immobilité, une fixité de celui qui commande et aussi de celui qui obéit. C'est qu'en effet la perpendicularité implique une position d'équilibre qui demande de peu dévier de son angle droit.

Il faudrait aussi évoquer l'intonation de la voix, qui n'est pas sans impliquer une verticalité liée à la vitesse de la diction de celui qui commande, sans cependant être empressée, pour donner cette impression d'immobilité. C'est donc plutôt l'attaque des mots qui est importante, c'est-à-dire une différentielle de vitesse par rapport à l'horizontalité du débit normal. J'appelle diagramme du commandement cette perpendicularité, elle suppose donc des conduites se référant à la verticale et à l'horizontale, ces conduites étant en relation. C'est cette mise en relation des conduites que j'appelle ici geste politique. Le geste du commandement s'exprime par un diagramme avec des intensités de direction topologiquement situées (de la verticale ou de l'horizontale). Dire que c'est l'action du commandant qui s'applique sur l'action du commandé, c'est donc mal dire, c'est plutôt le geste de commandement qui s'effectue 
par cette application coordonnée. Ce geste politique innerve donc la perception que nous avons des conduites de celui qui commande et de celui qui est commandé, d'un coup d'œil ou à l'oreille, nous savons si quelqu'un a de l'autorité ou pas, c'est-à-dire si quelqu'un a ou pas le diagramme en lui. Le geste de commander n'est donc pas accessoire, il ne suppose pas une signification qui le précèderait, il ne véhicule pas une information, c'est plutôt un performatif, effectuer un geste de commandement c'est comme le dire.

Autre exemple, le geste du biopouvoir actuel est celui qui implique un tri, une gestion sélective, or la perception des individus normalisés n'est pas sans être innervée par ce geste et donc par les affects de rejet de ceux qui ne sont pas sélectionnés, d'où le racisme. Il y a comme une grille perceptive liée au geste.

La perception de l'individu intégré totalement dans la communauté est en quelque mesure une semblable perception abstraite; au lieu de dégager l'objet du monde, elle découpe le monde selon des catégories qui correspondent aux classifications de la communauté, et établit entre les êtres des liens de participation affective selon ces catégories communautaires ${ }^{18}$.

29 Ou alors, le geste démocratique athénien est inséparable, selon Nicole Loraux, d'une relation d'actions opposées entre rivaux exprimant le geste de la stasis dont le diagramme est une symétrisation autour d'un milieu, le méson. Il y a "stasis en ce que l'affrontement à égalité des deux moitiés de la cité dresse dans le méson le conflit comme une stèle ». Le diagramme a donc trois places, celles symétriques des moitiés opposées et celle du milieu. Ainsi que des forces associées à celles-ci, forces opposées pour les places symétriques et forces compensées en ce centre d'indifférence qu'est la place du milieu, point d'ancrage virtuel de l'effectuation du geste. Il faudrait dire que les deux moitiés de la cité activent un diagramme qui s'effectue depuis leur milieu, le méson, elles sont sous l'horizon du geste qu'elles effectuent en l'activant à leurs places. On comprendra alors que le diagramme dans lequel on est situé politiquement est aussi en nous. Étant dans l'une des deux moitiés de la cité, je sens en moi l'opposition, je ne suis pas simplement une des actions opposées, et je fais l'expérience de la compensation, du point-milieu. C'est pourquoi Nicole Loraux peut dire que la rivalité maintient une certaine cohésion, une certaine unité de la cité, car elle est aussi éprouvée en nous. Le geste démocratique athénien innerve une certaine perception de l'égalité des rivaux (et de son point-milieu) comme celle, en mécanique, de forces opposées en équilibre. De même que l'objet perçu n'était pas séparable du geste, l'égalité est inséparable du geste, le geste n'est pas l'instrument d'une volonté d'égalité, il en est constitutif. Notre perception est tournée vers l'égalité, nous la voulons parce que nous suivons le geste démocratique. Notre volonté n'est que celle de la volition du geste.

30 Ce pourquoi un geste politique ne se définit jamais par son but, nos démocraties se tournent vers l'égalité tout à fait autrement que celle de la démocratie athénienne: elles réduisent les inégalités sous un geste de sélection, de compartimentation qui consiste à faire passer des traits dans un compartiment qui ne l'était pas avant (par exemple, le mariage dans le compartiment des homosexuels, un trait noir dans le compartiment présidentiel d'une case supposée blanche, comme dans le cas de l'élection d'Obama, etc.), ce pourquoi on criera à la victoire démocratique mais qui n'a rien à voir avec l'égalité des rivaux. 


\subsection{Retentissements politico-cinématographiques des gestes}

31 Pensons aussi à l'innervation gestuelle de la perception dans le cas d'un gesteévénement politique, un geste collectif comme peut l'être une émeute, où les gestes de chacun des acteurs du geste collectif font signe aux autres, et donc les perceptions aussi. Suivons Alain Brossat commentant un texte extrait du second volume de l'autobiographie de Canetti, Le Flambeau dans l'oreille :

L'événement que relate "L'Émeute " est l'incendie par une foule émeutière du Palais de Justice de Vienne, le 15 juillet 1927. Des ouvriers avaient été tués dans le Burgenland (territoire hongrois cédé à l'Autriche en 1920) au cours d'un conflit du travail, et le tribunal acquitta les meurtriers. Ce jugement déclencha l'émeute spontanée du 15 juillet à Vienne. La police tira dans le tas, faisant quatre-vingt-dix victimes ${ }^{19}$.

Le geste collectif de l'émeute est donc « incendier le Palais de Justice de Vienne ». Elias Canetti, témoin, raconte : «Un homme très grand, très fort, qui courait à côté de moi se frappa la poitrine du poing et hurla tout en courant: Ils tirent ! là, là. Et soudain, il disparut. Il n'était pas tombé. Où était-il ? » Ce geste insolite de se frapper la poitrine fait signe pour Canetti, si bien que, comme l'écrit Alain Brossat, il y a « un complet reconditionnement perceptif : c'est, déjà, dans l'ordre de la vision, de l'audition que s'inaugure le changement de la règle du jeu introduite par l'entrée dans le temps de la masse. Le geste le plus anodin devient signe de la rupture irrémédiable qui se produit alors $^{20} »$. Le geste collectif se diffracte donc en gestes multiples qui résonnent ou retentissent les uns dans les autres, c'est ce que j'appelle pour mon compte un champ gestuel. La perception est alors au plus proche du geste, ce sont des perceptions plus directes des gestes, comme si nous percevions avec le geste et non plus pour le servir (s'appliquant à sa part suffisante de conscience), d'où la pertinence de se référer aussi au rythme comme le fait Alain Brossat, le rythme étant à la fois geste et perception. "Ce rythme est ce qui assure la continuité de l'émeute en tant qu'événement et suite d'actions. On arrive donc à l'idée d'une musique, d'un environnement sonore qui inclut et ré-inclut sans fin l'individu dans l'espace de l'émeute ${ }^{21}{ }^{2}$.

Canetti va aussi parler d'une vague qui est comme le diagramme mobile de ce geste de l'émeute, composé de gestes individuels, de détails qui résonnent.

Cette journée, portée par un sentiment d'unité, fut une vague immense et unique qui déferla sur la ville et l'absorba ; quand la marée fut redescendue, on eut du mal à croire que la ville existait encore. Cette journée se composa de détails innombrables dont chacun se grava dans les mémoires, dont aucun ne fut oublié. Chacun d'eux est présent, aisément repérable et chacun constitue une part de cette vague gigantesque sans laquelle tout semble creux et absurde. Ce qu'il faudrait comprendre, ce serait la vague et non pas ses détails; j'ai souvent recommencé cette tentative pendant l'année qui suivit et plus tard encore, mais je n'y suis jamais parvenu.

La vague peut suggérer aussi que ce champ gestuel est plus virtualisant qu'actualisant, plus proche de l'enveloppe gestuelle, comme une vague au-dessus des actes massiques, en suspens.

Il y a donc ici comme un cinéma réel, vécu par les acteurs et Canetti, ce pourquoi Alain Brossat n'hésite pas à faire ce rapprochement. Le récit de Canetti :

se présente comme un dispositif d'enregistrement jeté au milieu de la foule, ou, si l'on veut, un appareil subjectif, un homme-œil, un homme-oreilles, un hommemuscles, un homme-poumons, etc. Bref, le dispositif qui se met en place ici apparaît 
de part en part cinématographique: la caméra-homme, jetée dans l'émeute, enregistre sans discontinuer images, sons, mouvements, puis des années plus tard, ces images et ces sons perdus-retrouvés sont montés par un documentaristeécrivain (également connu sous le nom de Canetti...) convaincu que ce fonds constitue un trésor susceptible de livrer la clé historique $\mathrm{du} \mathrm{xx}^{\mathrm{e}}$ siècle.... ${ }^{22}$

Si j'ai pu dire tout à l'heure que le cinéma avait des retombées politiques, on voit maintenant qu'à l'inverse, il y a comme une cinématographie propre à certains gestes politiques. Réalité et cinéma convergent. Il y a un cinéma de la réalité. Disons alors pour terminer, et sous forme d'une seule suggestion, que cela se rapproche d'une autre intuition de Benjamin : la seconde technique dont fait partie le cinéma renvoie à une harmonie de la nature et de l'humanitér3.

\section{BIBLIOGRAPHIE}

Benjamin Walter, L'œuvre d'art à l'époque de sa reproductibilité technique, Lionel Duvoy (trad.), Paris, Allia, 2003.

Benjamin Walter, Écrits français, Paris, Gallimard, 1991.

Benjamin Walter, « Problèmes de sociologie du langage », Maurice de Gandillac (trad.), revue par Pierre Rusch, dans Euvres III, Paris, Gallimard, 2000.

Benjamin Walter, «L'œuvre d'art à l'ère de la reproductibilité technique », Rainer Rochlitz (trad.), dans Euvres III, Paris, Gallimard, 2000.

Berthoz Alain, Petit Jean-Luc, Phénoménologie et physiologie de l'action, Paris, Odile Jacob, 2006.

Brossat Alain, Ce qui fait époque, Paris, L'Harmattan (Esthétiques), 2007.

Citton Yves, Gestes d'humanités, Paris, A. Colin, 2012.

Citton Yves, Renverser l'insoutenable, Paris, Seuil, 2012.

Naze Alain, Temps, récit et transmission chez W. Benjamin et P. P. Pasolini, Paris, L'Harmattan (Esthétiques), t. I, 2011.

Nietzsche Friedrich, La Généalogie de la morale, Paris, Flammarion, 2002.

Olivier Gérard, La Cognition gestuelle, Grenoble, Presses universitaires de Grenoble, 2012.

Simondon Gilbert, L'individuation psychique et collective, Paris, Aubier, 2007.

Spinoza Baruch, Éthique, Robert Misrahi (trad.), Paris, Éditions de l'éclat, 2005.

\section{NOTES}

1. Friedrich Nietzsche, La Généalogie de la morale, Paris, Flammarion, 2002.

2. Baruch Spinoza, Éthique, Robert Misrahi (trad.), Paris, Éditions de l'éclat, 2005, p. 117.

3. Ibid., proposition 40, V.

4. Yves Citton, Renverser l'insoutenable, Paris, Seuil, 2012, p. 146. 
5. Alain Berthoz, Jean-Luc Petit, Phénoménologie et physiologie de l'action, Paris, Odile Jacob, 2006, p. 71.

6. Ibid.

7. Gérard Olivier, La Cognition gestuelle, Grenoble, Presses universitaires de Grenoble, 2012, p. 58.

8. Ibid., p. 74.

9. Ibid., p. 75.

10. Walter Benjamin, «Problèmes de sociologie du langage », Maurice de Gandillac (trad.), revue par Pierre Rusch, dans Euvres III, Paris, Gallimard, 2000, p. 40.

11. Walter Benjamin, "L'œuvre d'art à l'ère de la reproductibilité technique ", Rainer Rochlitz (trad.), dans Euvres III, p. 103.

12. Gérard Olivier, La Cognition gestuelle, p. 45.

13. Ibid., p. 49.

14. Walter Benjamin, L'œuvre d'art à l'époque de sa reproductibilité technique, Lionel Duvoy (trad.), Paris, Allia, 2003, note 1, p. 54.

15. Alain Naze, Temps, récit et transmission chez W. Benjamin et P. P. Pasolini, Paris, L'Harmattan, t. I, 2011, p. 199.

16. Ibid., p. 198.

17. Yves Citton, Gestes d'humanités, Paris, A. Colin, 2012.

18. Gilbert Simondon, L'individuation psychique et collective, Paris, Aubier, 2007, p. 288.

19. Alain Brossat, Ce qui fait époque, Paris, L'Harmattan, 2007, p. 151. Alain Brossat nous rapporte qu'un des biographes de Canetti pense que cet événement a été déterminant : " pour l'essentiel, son destin et son œuvre se décidèrent ce jour-là, devant les morts et les flammes ».

20. Ibid., p. 157-158.

21. Ibid., p. 158.

22. Ibid., p. 156.

23. Walter Benjamin, Écrits français, Paris, Gallimard, 1991, p. 189.

\section{RÉSUMÉS}

Walter Benjamin fait usage du concept d'innervation qu'il a trouvé chez Fiedler. On pourrait dire que, par innervation, on entend le fait d'actionner les nerfs moteurs ou sensitifs. Benjamin semble ne le réserver qu'aux nerfs moteurs innervés par les appareils. Je voudrais pour mon compte montrer que le geste innerve autant nos perceptions que nos actions et que cela peut même être pensé en prenant appui sur Benjamin (mais aussi sur la neurophysiologie). Je viserai plus particulièrement les champs politique et cinématographique, qui ne sont pas sans intersections.

\section{INDEX}

Mots-clés : innervation, geste, cinéma, politique, perception, diagramme, neurophysiologie, virtuel

Personnes citées : Benjamin (Walter) 


\section{AUTEUR}

\section{PHILIPPE ROY}

Philippe Roy est enseignant en philosophie, doctorant en philosophie à l'université de Paris 8. Il a publié Tombeau pour Pierre Rivière, Paris, L'Harmattan, 2013 et Trouer la membrane. Penser et vivre la politique par des gestes, Paris, L'Harmattan, 2012. 